\title{
Numerical and Statistical Quantifications of Biodiversity: Two-At-A-Time Equal Variations
}

\author{
Ekaka-a E.N. ${ }^{*}$, Osahogulu D.J. ${ }^{2}$, Atsu J. U. ${ }^{3 *}$, Isibor L. A. ${ }^{2}$ \\ ${ }^{1}$ Department of Mathematics Rivers State University, Nkpolu, Port Harcourt, Nigeria \\ ${ }^{2}$ Department of Mathematics/Statistics, Ignatius Ajuru University of Education, Rumuolumeni, Port Harcourt, Nigeria \\ ${ }^{3}$ Department of Mathematics/Statistics, Cross River State University of Technology, Calabar, Nigeria
}

\begin{abstract}
The ecological concept of biodiversity is a challenging environmental problem that requires a sound mathematical reasoning. We have used the method of a numerical simulation that is indexed by a numerical scheme to predict biodiversity loss and biodiversity gain due to a decreasing and increasing variations of the intrinsic growth rates together. The novel results that we have obtained that we have not seen elsewhere, but do complement other similar numerical predictions of biodiversity are presented and discussed quantitatively.
\end{abstract}

Keywords - Ecological concept, environmental problem, biodiversity, numerical simulation, intrinsic growth rate, ecosystem stability.

\section{INTRODUCTION}

The ongoing debate between biodiversity, ecosystem stability, and its implications, Atsu and Ekaka-a( 2017) ${ }^{1}$ makes it imperative to examine the effects of varying the intrinsic growth rates together on biodiversity loss and biodiversity gain by using a computationally efficient numerical scheme called Matlab function ordinary differential equation of order 45 (ODE 45). Other related contributions on the link between biodiversity and ecosystem stability have been adequately sighted. ([2] - [26]).

\section{MATERIALS AND METHODS}

If a variation of a model parameter value produces a new biomass which is smaller than the old biomass for any interacting legumes, such as cowpea and groundnut, then a biodiversity loss has occurred and can be quantified as we have done in this study.

On the other hand, if a variation of a model parameter value produces a new biomass which outweighs the old biomass irrespective of the type of legumes, then a biodiversity gain has occurred and can be similarly quantified.

Following Ekaka-a et al (2009), we have considered the following continuous dynamical system of nonlinear first order ordinary differential equation

$$
\begin{aligned}
& \frac{d}{d t} \mathrm{C}(\mathrm{t})=\alpha_{1} \mathrm{C}(\mathrm{t})-\beta_{1} \mathrm{C}^{2}(\mathrm{t})-\mathrm{r}_{1} \mathrm{C}(\mathrm{t}) \mathrm{G}(\mathrm{t}) \\
& \frac{d}{d t} \mathrm{G}(\mathrm{t})=\alpha_{2} \mathrm{G}(\mathrm{t})-\beta_{2} \mathrm{G}^{2}(\mathrm{t})-\mathrm{r}_{2} \mathrm{C}(\mathrm{t}) \mathrm{G}(\mathrm{t}) \\
& \text { With } \mathrm{C}(0)=0.12 \quad \text { and } \quad \mathrm{G}(0)=0.14
\end{aligned}
$$

For the purpose of clarity, the variables and the parameter values for these model equations are defined as follows

- $\mathrm{C}_{b}(\mathrm{t})$ and $\mathrm{G}_{b}(\mathrm{t})$ are called the biomass of cowpea and groundnut at time (t) in the unit of weeks

- $\alpha_{1}$ and $\alpha_{2}$ are called the intrinsic growth rates for populations $\mathrm{C}_{b}(\mathrm{t})$ and $\mathrm{G}_{b}(\mathrm{t})$ in the absence of selfinteraction and inter-competition interaction

- $\quad \beta_{1}$ and $\beta_{2}$ are called the intra-competition coefficients

- $\mathrm{r}_{1}$ and $\mathrm{r}_{2}$ are called the inter-competition coefficients to analyze our propose problem,

$\alpha_{1}=0.0225, \alpha_{2}=0.0446 ; \beta_{1}=0.0069, \beta_{2}=0.0133 ; r_{1}=$ $0.0018, \mathrm{r}_{2}=0.0012$.

The core numerical method that we have used in this present analysis is called ODE 45 .

\section{RESULTS AND DISCUSSIONS}

The results of this study are displayed and discussed quantitatively in Tables 1.1, 1.2, 1.3, 1.4, 1.5, 2.1, 2.2, 2.3, 2.4 and 2.5 .

Table.1.1: Evaluating the effect of varying the intrinsic

\begin{tabular}{|c|c|c|c|c|c|}
\hline$C_{b}(t)$ & $C_{b m}(t) B$ & BL(\%) & $\mathbf{G b}(\mathbf{t})$ & $G_{b m}(t) \quad B$ & $\mathrm{~L}(\%)$ \\
\hline 0.1200 & 0.1200 & 0 & 0.1200 & 0.1200 & 0 \\
\hline 0.1226 & 0.1201 & 2.0034 & 0.1253 & 0.1203 & 3.9312 \\
\hline 0.1253 & 0.1203 & 3.9641 & 0.1307 & 0.1207 & 7.7015 \\
\hline 0.1280 & 0.1204 & 5.8831 & 0.1364 & 0.1210 & 11.3173 \\
\hline 0.1307 & 0.1206 & 7.7611 & 0.1424 & 0.1213 & 14.7850 \\
\hline 0.1335 & 0.1207 & 9.5991 & 0.1485 & 0.1216 & 18.1105 \\
\hline 0.1364 & 0.1209 & 11.3979 & 0.1550 & 0.1220 & 21.2998 \\
\hline 0.1393 & 0.1210 & 13.1582 & 0.1617 & 0.1223 & 24.3584 \\
\hline 0.1423 & 0.1212 & 14.8809 & 0.1686 & 0.1226 & 27.2915 \\
\hline
\end{tabular}
growth rates together by $10 \%$ on biodiversity loss using ODE 45 numerical scheme. 


\begin{tabular}{|llllll|}
\hline 0.1454 & 0.1213 & 16.5668 & 0.1759 & 0.1230 & 30.1044 \\
\hline 0.1485 & 0.1214 & 18.2166 & 0.1835 & 0.1233 & 32.8018 \\
\hline 0.1517 & 0.1216 & 19.8310 & 0.1913 & 0.1236 & 35.3886 \\
\hline 0.1549 & 0.1217 & 21.4108 & 0.1995 & 0.1239 & 37.8693 \\
\hline 0.1582 & 0.1219 & 22.9568 & 0.2080 & 0.1243 & 40.2481 \\
\hline 0.1616 & 0.1220 & 24.4695 & 0.2168 & 0.1246 & 42.5293 \\
\hline 0.1650 & 0.1222 & 25.9497 & 0.2260 & 0.1249 & 44.7168 \\
\hline 0.1685 & 0.1223 & 27.3980 & 0.2355 & 0.1253 & 46.8144 \\
\hline 0.1720 & 0.1225 & 28.8152 & 0.2454 & 0.1256 & 48.8258 \\
\hline 0.1757 & 0.1226 & 30.2018 & 0.2557 & 0.1259 & 50.7546 \\
\hline 0.1793 & 0.1227 & 31.5585 & 0.2664 & 0.1263 & 52.6041 \\
\hline
\end{tabular}

From Table 1.1, when all the model parameter values are fixed, the cowpea biomass data denoted $\mathrm{C}_{\mathrm{b}}(\mathrm{t})$ when the length of the growing season is twenty one weeks range from a low value of 0.12 grams/area to 0.1793 grams/area whereas $\mathrm{C}_{\mathrm{bm}}(\mathrm{t})$ data range from a low value 0.12 grams/area to 0.1227 grams/area due to a $10 \%$ variation of the intrinsic growth rates together. On the basis of this calculation, the new simulated cowpea data due to a joint variation of the intrinsic growth rates dominantly predicts a depletion which mimics biodiversity loss. The extent of biodiversity loss has been quantified to range from zero to 31.6 approximately providing an average of 16.8 which re-classifies the vulnerability of the cowpea biomass to biodiversity loss. A similar observation can be made from the groundnut biomass component. In summary, the groundnut biomass is about 1.67 approximately more vulnerable to biodiversity loss than the cowpea biomass. Statistically, the average of biomass vulnerability to biodiversity loss with respect to the groundnut legume is $29.57 \%$ approximately.

Table.1.2: Evaluating the effect of varying the intrinsic growth rates together by $15 \%$ on biodiversity loss using ODE 45 numerical scheme.

\begin{tabular}{|c|c|c|c|c|c|}
\hline$C_{b}(t)$ & $\mathrm{C}_{\mathrm{bm}}(\mathrm{t}) \mathrm{BI}$ & $\mathrm{L}(\%)$ & $\mathbf{G}_{b}(\mathbf{t})$ & $\mathbf{G}_{b m}(\mathbf{t})$ & BL(\%) \\
\hline 0.1200 & 0.1200 & 0 & 0.1200 & 0.1200 & 0 \\
\hline 0.1226 & 0.1203 & 1.8931 & 0.1253 & 0.1206 & 3.7169 \\
\hline 0.1253 & 0.1206 & 3.7480 & 0.1307 & 0.1212 & 7.2896 \\
\hline 0.1280 & 0.1208 & 5.5655 & 0.1364 & 0.1218 & 10.7235 \\
\hline 0.1307 & 0.1211 & 7.3462 & 0.1424 & 0.1224 & 14.0240 \\
\hline 0.1335 & 0.1214 & 9.0908 & 0.1485 & 0.1230 & 17.1963 \\
\hline 0.1364 & 0.1217 & 10.8001 & 0.1550 & 0.1236 & 20.2451 \\
\hline 0.1393 & 0.1220 & 12.4747 & 0.1617 & 0.1242 & 23.1754 \\
\hline 0.1423 & 0.1222 & 14.1153 & 0.1686 & 0.1248 & 25.9917 \\
\hline 0.1454 & 0.1225 & 15.7226 & 0.1759 & 0.1254 & 28.6983 \\
\hline
\end{tabular}

\begin{tabular}{|llllll|}
\hline 0.1485 & 0.1228 & 17.2972 & 0.1835 & 0.1260 & 31.2995 \\
\hline 0.1517 & 0.1231 & 18.8398 & 0.1913 & 0.1267 & 33.7993 \\
\hline 0.1549 & 0.1234 & 20.3509 & 0.1995 & 0.1273 & 36.2017 \\
\hline 0.1582 & 0.1237 & 21.8312 & 0.2080 & 0.1279 & 38.5104 \\
\hline 0.1616 & 0.1239 & 23.2813 & 0.2168 & 0.1285 & 40.7289 \\
\hline 0.1650 & 0.1242 & 24.7017 & 0.2260 & 0.1291 & 42.8609 \\
\hline 0.1685 & 0.1245 & 26.0930 & 0.2355 & 0.1298 & 44.9095 \\
\hline 0.1720 & 0.1248 & 27.4558 & 0.2454 & 0.1304 & 46.8781 \\
\hline 0.1757 & 0.1251 & 28.7906 & 0.2557 & 0.1310 & 48.7696 \\
\hline 0.1793 & 0.1254 & 30.0981 & 0.2664 & 0.1316 & 50.5872 \\
\hline
\end{tabular}

From Table 1.2, when all the model parameter values are fixed, the cowpea biomass data denoted $\mathrm{C}_{b}(\mathrm{t})$ when the length of the growing season is twenty one weeks range from a low value of 0.12 grams/area to 0.1793 grams/area whereas $\mathrm{C}_{\mathrm{bm}}(\mathrm{t})$ data range from a low value 0.12 grams/area to 0.1254 grams/area due to a $15 \%$ variation of the intrinsic growth rates together. On the basis of this calculation, the new simulated cowpea data due to a joint variation of the intrinsic growth rates dominantly predicts a depletion which mimics biodiversity loss. The extent of biodiversity loss has been quantified to range from zero to 30.1 approximately providing an average of 15.97 which re-classifies the vulnerability of the cowpea biomass to biodiversity loss. A similar observation can be made from the groundnut biomass component. In summary, the groundnut biomass is about 1.68 approximately more vulnerable to biodiversity loss than the cowpea biomass. Statistically, the average of biomass vulnerability to biodiversity loss with respect to the groundnut legume is $28.28 \%$ approximately.

Table.1.3: Evaluating the effect of varying the intrinsic growth rates together by $20 \%$ on biodiversity loss using ODE 45 numerical scheme.

\begin{tabular}{|cccccc|}
\hline $\mathbf{C}_{\mathbf{b}}(\mathbf{t})$ & \multicolumn{2}{c}{$\mathbf{C}_{\mathbf{b m}}(\mathbf{t}) \mathbf{B L}(\boldsymbol{\%})$} & $\mathbf{G}_{\mathbf{b}}(\mathbf{t})$ & $\mathbf{G}_{\mathbf{b m}}(\mathbf{t})$ & $\mathbf{B L}(\boldsymbol{\%})$ \\
\hline 0.1200 & 0.1200 & 0 & 0.1200 & 0.1200 & 0 \\
\hline 0.1226 & 0.1204 & 1.7828 & 0.1253 & 0.1209 & 3.5022 \\
\hline 0.1253 & 0.1208 & 3.5315 & 0.1307 & 0.1217 & 6.8759 \\
\hline 0.1280 & 0.1212 & 5.2468 & 0.1364 & 0.1226 & 10.1258 \\
\hline 0.1307 & 0.1217 & 6.9293 & 0.1424 & 0.1235 & 13.2563 \\
\hline 0.1335 & 0.1221 & 8.5796 & 0.1485 & 0.1244 & 16.2718 \\
\hline 0.1364 & 0.1225 & 10.1982 & 0.1550 & 0.1253 & 19.1764 \\
\hline 0.1393 & 0.1229 & 11.7858 & 0.1617 & 0.1261 & 21.9741 \\
\hline 0.1423 & 0.1233 & 13.3429 & 0.1686 & 0.1270 & 24.6688 \\
\hline 0.1454 & 0.1238 & 14.8699 & 0.1759 & 0.1279 & 27.2641 \\
\hline 0.1485 & 0.1242 & 16.3676 & 0.1835 & 0.1289 & 29.7638 \\
\hline
\end{tabular}




\begin{tabular}{|llllll|}
\hline 0.1517 & 0.1246 & 17.8364 & 0.1913 & 0.1298 & 32.1712 \\
\hline 0.1549 & 0.1250 & 19.2768 & 0.1995 & 0.1307 & 34.4897 \\
\hline 0.1582 & 0.1255 & 20.6893 & 0.2080 & 0.1316 & 36.7225 \\
\hline 0.1616 & 0.1259 & 22.0745 & 0.2168 & 0.1325 & 38.8727 \\
\hline 0.1650 & 0.1263 & 23.4328 & 0.2260 & 0.1335 & 40.9432 \\
\hline 0.1685 & 0.1267 & 24.7647 & 0.2355 & 0.1344 & 42.9371 \\
\hline 0.1720 & 0.1272 & 26.0707 & 0.2454 & 0.1353 & 44.8570 \\
\hline 0.1757 & 0.1276 & 27.3513 & 0.2557 & 0.1363 & 46.7055 \\
\hline 0.1793 & 0.1280 & 28.6068 & 0.2664 & 0.1372 & 48.4854 \\
\hline
\end{tabular}

\begin{tabular}{|llllll|}
\hline 0.1582 & 0.1273 & 19.5309 & 0.2080 & 0.1354 & 34.8830 \\
\hline 0.1616 & 0.1279 & 20.8489 & 0.2168 & 0.1367 & 36.9588 \\
\hline 0.1650 & 0.1284 & 22.1427 & 0.2260 & 0.1379 & 38.9619 \\
\hline 0.1685 & 0.1290 & 23.4128 & 0.2355 & 0.1392 & 40.8947 \\
\hline 0.1720 & 0.1296 & 24.6594 & 0.2454 & 0.1405 & 42.7597 \\
\hline 0.1757 & 0.1302 & 25.8831 & 0.2557 & 0.1418 & 44.5592 \\
\hline 0.1793 & 0.1308 & 27.0841 & 0.2664 & 0.1431 & 46.2954 \\
\hline
\end{tabular}

From Table 1.3, when all the model parameter values are fixed, the cowpea biomass data $\mathrm{C}_{b}(\mathrm{t})$ when the length of the growing season is twenty one weeks range from a low value of 0.12 grams/area to 0.1793 grams/area whereas $\mathrm{C}_{\mathrm{bm}}(\mathrm{t})$ data range from a low value 0.12 grams/area to 0.1280 grams/area due to a $20 \%$ variation of the intrinsic growth rates together. On the basis of this calculation, the new simulated cowpea data due to a joint variation of the intrinsic growth rates dominantly predicts a depletion which mimics biodiversity loss. The extent of biodiversity loss has been quantified to range from zero to 28.6 approximately providing an average of 15.14 which re-classifies the vulnerability of the cowpea biomass to biodiversity loss. A similar observation can be made from the groundnut biomass component. In summary, the groundnut biomass is about 1.69 approximately more vulnerable to biodiversity loss than the cowpea biomass. Statistically, the average of biomass vulnerability to biodiversity loss with respect to the groundnut legume is $26.95 \%$ approximately.

Table.1.4: Evaluating the effect of varying the intrinsic growth rates together by $25 \%$ on biodiversity loss using ODE 45 numerical scheme.

\begin{tabular}{|ccrcccc|}
\hline $\mathrm{C}_{\mathrm{b}}(\mathrm{t})$ & \multicolumn{2}{c}{$\mathrm{C}_{\mathrm{bm}}(\mathrm{t}) \mathrm{BL}(\%)$} & $\mathrm{G}_{\mathrm{b}}(\mathrm{t})$ & \multicolumn{2}{c|}{$\mathrm{G}_{\mathrm{bm}}(\mathrm{t})$} & \multicolumn{2}{c|}{$\mathrm{BL}(\%)$} \\
\hline 0.1200 & 0.1200 & 0 & 0.1200 & 0.1200 & 0 \\
\hline 0.1226 & 0.1206 & 1.6723 & 0.1253 & 0.1211 & 3.2869 \\
\hline 0.1253 & 0.1211 & 3.3145 & 0.1307 & 0.1223 & 6.4603 \\
\hline 0.1280 & 0.1217 & 4.9271 & 0.1364 & 0.1234 & 9.5240 \\
\hline 0.1307 & 0.1222 & 6.5106 & 0.1424 & 0.1246 & 12.4818 \\
\hline 0.1335 & 0.1228 & 8.0656 & 0.1485 & 0.1258 & 15.3370 \\
\hline 0.1364 & 0.1233 & 9.5924 & 0.1550 & 0.1269 & 18.0933 \\
\hline 0.1393 & 0.1239 & 11.0915 & 0.1617 & 0.1281 & 20.7540 \\
\hline 0.1423 & 0.1245 & 12.5635 & 0.1686 & 0.1293 & 23.3223 \\
\hline 0.1454 & 0.1250 & 14.0087 & 0.1759 & 0.1305 & 25.8013 \\
\hline 0.1485 & 0.1256 & 15.4276 & 0.1835 & 0.1317 & 28.1940 \\
\hline 0.1517 & 0.1262 & 16.8207 & 0.1913 & 0.1330 & 30.5033 \\
\hline 0.1549 & 0.1267 & 18.1883 & 0.1995 & 0.1342 & 32.7321 \\
\hline
\end{tabular}

Table.1.5: Evaluating the effect of varying the intrinsic growth rates together by $95 \%$ on biodiversity loss using ODE 45 numerical scheme.

\begin{tabular}{|c|c|c|c|c|c|}
\hline $\mathrm{C}_{\mathrm{b}}(\mathrm{t})$ & $\mathrm{C}_{\mathrm{bm}}(\mathrm{t}) \mathrm{BL}$ & $(\%)$ & $\mathrm{J}_{\mathrm{b}}(\mathrm{t})$ & $\mathrm{G}_{\mathrm{bm}}(\mathrm{t})$ & $3 \mathrm{~L}(\%)$ \\
\hline 0.1200 & 0.1200 & 0 & 0.1200 & 0.1200 & 0 \\
\hline 0.1226 & 0.1225 & 0.1124 & 0.1253 & 0.1250 & 0.2226 \\
\hline 0.1253 & 0.1250 & 0.2245 & 0.1307 & 0.1301 & 0.4442 \\
\hline 0.1280 & 0.1275 & 0.3363 & 0.1364 & 0.1355 & 0.6650 \\
\hline 0.1307 & 0.1301 & 0.4478 & 0.1424 & 0.1411 & 0.8847 \\
\hline 0.1335 & 0.1328 & 0.5590 & 0.1485 & 0.1469 & 1.1035 \\
\hline 0.1364 & 0.1355 & 0.6699 & 0.1550 & 0.1529 & 1.3213 \\
\hline 0.1393 & 0.1383 & 0.7805 & 0.1617 & 0.1592 & 1.5381 \\
\hline 0.1423 & 0.1411 & 0.8908 & 0.1686 & 0.1657 & 1.7537 \\
\hline 0.1454 & 0.1439 & 1.0007 & 0.1759 & 0.1724 & 1.9682 \\
\hline 0.1485 & 0.1468 & 1.1103 & 0.1835 & 0.1795 & 2.1815 \\
\hline 0.1517 & 0.1498 & 1.2195 & 0.1913 & 0.1867 & 2.3936 \\
\hline 0.1549 & 0.1528 & 1.3284 & 0.1995 & 0.1943 & 2.6045 \\
\hline 0.1582 & 0.1559 & 1.4369 & 0.2080 & 0.2021 & 2.8141 \\
\hline 0.1616 & 0.1591 & 1.5450 & 0.2168 & 0.2103 & 3.0223 \\
\hline 0.1650 & 0.1623 & 1.6527 & 0.2260 & 0.2187 & 3.2291 \\
\hline 0.1685 & 0.1655 & 1.7600 & 0.2355 & 0.2274 & 3.4344 \\
\hline 0.1720 & 0.1688 & 1.8668 & 0.2454 & 0.2365 & 3.6383 \\
\hline 0.1757 & 0.1722 & 1.9733 & 0.2557 & 0.2459 & 3.8406 \\
\hline 0.1793 & 0.1756 & 2.0792 & 0.2664 & 0.2556 & 4.0412 \\
\hline
\end{tabular}

Table.2.1: Evaluating the effect of varying the intrinsic growth rates together by $105 \%$ on biodiversity gain using ODE 45 numerical scheme.

\begin{tabular}{|rcccccc|}
\hline $\mathrm{C}_{\mathrm{b}}(\mathrm{t})$ & \multicolumn{2}{c|}{$\mathrm{C}_{\mathrm{bm}}(\mathrm{t}) \mathrm{BG}(\%)$} & $\mathrm{G}_{\mathrm{b}}(\mathrm{t})$ & $\mathrm{G}_{\mathrm{bm}}(\mathrm{t})$ & \multicolumn{2}{c|}{$\mathrm{BG}(\%)$} \\
\hline 0.1200 & 0.1200 & 0 & 0.1200 & 0.1200 & 0 \\
\hline 0.1226 & 0.1227 & 0.1125 & 0.1253 & 0.1255 & 0.2231 \\
\hline 0.1253 & 0.1255 & 0.2250 & 0.1307 & 0.1313 & 0.4462 \\
\hline 0.1280 & 0.1284 & 0.3374 & 0.1364 & 0.1373 & 0.6694 \\
\hline 0.1307 & 0.1313 & 0.4498 & 0.1424 & 0.1436 & 0.8926 \\
\hline 0.1335 & 0.1343 & 0.5621 & 0.1485 & 0.1502 & 1.1158 \\
\hline 0.1364 & 0.1373 & 0.6744 & 0.1550 & 0.1570 & 1.3389 \\
\hline
\end{tabular}




\begin{tabular}{|llllll|}
\hline 0.1393 & 0.1404 & 0.7866 & 0.1617 & 0.1642 & 1.5620 \\
\hline 0.1423 & 0.1436 & 0.8987 & 0.1686 & 0.1717 & 1.7848 \\
\hline 0.1454 & 0.1469 & 1.0108 & 0.1759 & 0.1794 & 2.0074 \\
\hline 0.1485 & 0.1502 & 1.1227 & 0.1835 & 0.1875 & 2.2298 \\
\hline 0.1517 & 0.1535 & 1.2345 & 0.1913 & 0.1960 & 2.4518 \\
\hline 0.1549 & 0.1570 & 1.3461 & 0.1995 & 0.2048 & 2.6735 \\
\hline 0.1582 & 0.1605 & 1.4576 & 0.2080 & 0.2140 & 2.8947 \\
\hline 0.1616 & 0.1641 & 1.5690 & 0.2168 & 0.2236 & 3.1153 \\
\hline 0.1650 & 0.1678 & 1.6801 & 0.2260 & 0.2335 & 3.3354 \\
\hline 0.1685 & 0.1715 & 1.7911 & 0.2355 & 0.2439 & 3.5548 \\
\hline 0.1720 & 0.1753 & 1.9018 & 0.2454 & 0.2547 & 3.7734 \\
\hline 0.1757 & 0.1792 & 2.0124 & 0.2557 & 0.2659 & 3.9912 \\
\hline 0.1793 & 0.1832 & 2.1226 & 0.2664 & 0.2776 & 4.2080 \\
\hline
\end{tabular}

Table.2.2: Evaluating the effect of varying the intrinsic growth rates together by $110 \%$ on biodiversity gain using ODE 45 numerical scheme.

\begin{tabular}{|c|c|c|c|c|c|}
\hline $\mathrm{C}_{\mathrm{b}}(\mathrm{t})$ & $\mathrm{C}_{\mathrm{bm}}(\mathrm{t}) \mathrm{B}$ & $\mathrm{G}(\%)$ & $\mathrm{G}_{\mathrm{b}}(\mathrm{t})$ & $\mathrm{G}_{\mathrm{bm}}(\mathrm{t})$ & $\mathrm{BG}(\%)$ \\
\hline 0.1200 & 0.1200 & 0 & 0.1200 & 0.1200 & 0 \\
\hline 0.1226 & 0.1229 & 0.2251 & 0.1253 & 0.1258 & 0.4466 \\
\hline 0.1253 & 0.1258 & 0.4504 & 0.1307 & 0.1319 & 0.8944 \\
\hline 0.1280 & 0.1288 & 0.6759 & 0.1364 & 0.1383 & 1.3433 \\
\hline 0.1307 & 0.1319 & 0.9016 & 0.1424 & 0.1449 & 1.7932 \\
\hline 0.1335 & 0.1350 & 1.1274 & 0.1485 & 0.1519 & 2.2441 \\
\hline 0.1364 & 0.1383 & 1.3533 & 0.1550 & 0.1592 & 2.6958 \\
\hline 0.1393 & 0.1415 & 1.5794 & 0.1617 & 0.1668 & 3.1482 \\
\hline 0.1423 & 0.1449 & 1.8055 & 0.1686 & 0.1747 & 3.6013 \\
\hline 0.1454 & 0.1483 & 2.0317 & 0.1759 & 0.1830 & 4.0549 \\
\hline 0.1485 & 0.1518 & 2.2578 & 0.1835 & 0.1917 & 4.5089 \\
\hline 0.1517 & 0.1554 & 2.4840 & 0.1913 & 0.2008 & 4.9632 \\
\hline 0.1549 & 0.1591 & 2.7102 & 0.1995 & 0.2103 & 5.4177 \\
\hline 0.1582 & 0.1628 & 2.9363 & 0.2080 & 0.2202 & 5.8722 \\
\hline 0.1616 & 0.1667 & 3.1623 & 0.2168 & 0.2305 & 6.3265 \\
\hline 0.1650 & 0.1706 & 3.3881 & 0.2260 & 0.2413 & 6.7804 \\
\hline 0.1685 & 0.1746 & 3.6138 & 0.2355 & 0.2526 & 7.2339 \\
\hline 0.1720 & 0.1786 & 3.8393 & 0.2454 & 0.2643 & 7.6867 \\
\hline 0.1757 & 0.1828 & 4.0646 & 0.2557 & 0.2765 & 8.1387 \\
\hline 0.1793 & 0.1870 & 4.2895 & 0.2664 & 0.2893 & 8.5895 \\
\hline
\end{tabular}

Table.2.3: Evaluating the effect of varying the intrinsic growth rates together by $115 \%$ on biodiversity gain using ODE 45 numerical scheme.

\begin{tabular}{|c|c|c|c|c|c|}
\hline $\mathrm{C}_{\mathrm{b}}(\mathrm{t})$ & \multicolumn{2}{|c|}{$\mathrm{C}_{\mathrm{bm}}(\mathrm{t}) \mathrm{BG}(\%)$} & $\mathrm{G}_{\mathrm{b}}(\mathrm{t})$ & $\mathrm{G}_{\mathrm{bm}}(\mathrm{t})$ & $\mathrm{BG}(\%)$ \\
\hline 0.1200 & 0.1200 & 0 & 0.1200 & 0.1200 & 0 \\
\hline 0.1226 & 0.1230 & 0.3379 & 0.1253 & 0.1261 & 0.6707 \\
\hline 0.1253 & 0.1261 & 0.6764 & 0.1307 & 0.1325 & 1.3446 \\
\hline 0.1280 & 0.1293 & 1.0156 & 0.1364 & 0.1392 & 2.0217 \\
\hline 0.1307 & 0.1325 & 1.3554 & 0.1424 & 0.1462 & 2.7018 \\
\hline 0.1335 & 0.1358 & 1.6959 & 0.1485 & 0.1536 & 3.3848 \\
\hline 0.1364 & 0.1392 & 2.0368 & 0.1550 & 0.1613 & 4.0707 \\
\hline 0.1393 & 0.1427 & 2.3784 & 0.1617 & 0.1694 & 4.7591 \\
\hline 0.1423 & 0.1462 & 2.7204 & 0.1686 & 0.1778 & 5.4500 \\
\hline 0.1454 & 0.1498 & 3.0628 & 0.1759 & 0.1867 & 6.1432 \\
\hline 0.1485 & 0.1536 & 3.4057 & 0.1835 & 0.1960 & 6.8385 \\
\hline 0.1517 & 0.1574 & 3.7489 & 0.1913 & 0.2057 & 7.5357 \\
\hline 0.1549 & 0.1612 & 4.0924 & 0.1995 & 0.2159 & 8.2345 \\
\hline 0.1582 & 0.1652 & 4.4362 & 0.2080 & 0.2266 & 8.9348 \\
\hline 0.1616 & 0.1693 & 4.7803 & 0.2168 & 0.2377 & 9.6363 \\
\hline 0.1650 & 0.1734 & 5.1244 & 0.2260 & 0.2494 & 10.3387 \\
\hline 0.1685 & 0.1777 & 5.4687 & 0.2355 & 0.2615 & 11.0417 \\
\hline 0.1720 & 0.1820 & 5.8130 & 0.2454 & 0.2743 & 11.7451 \\
\hline 0.1757 & 0.1865 & 6.1573 & 0.2557 & 0.2875 & 12.4484 \\
\hline 0.1793 & 0.1910 & 6.5016 & 0.2664 & 0.3014 & 13.1514 \\
\hline
\end{tabular}

Table.2.4: Evaluating the effect of varying the intrinsic growth rates together by $120 \%$ on biodiversity gain using ODE 45 numerical scheme.

\begin{tabular}{|ccccccc|}
\hline $\mathrm{C}_{\mathrm{b}}(\mathrm{t})$ & \multicolumn{2}{c}{$\mathrm{C}_{\mathrm{bm}}(\mathrm{t}) \mathrm{BG}(\%)$} & $\mathrm{G}_{\mathrm{b}}(\mathrm{t})$ & \multicolumn{2}{c|}{$\mathrm{G}_{\mathrm{bm}}(\mathrm{t})$} & $\mathrm{BG}(\%)$ \\
\hline 0.1200 & 0.1200 & 0 & 0.1200 & 0.1200 & 0 \\
\hline 0.1226 & 0.1232 & 0.4507 & 0.1253 & 0.1264 & 0.8952 \\
\hline 0.1253 & 0.1264 & 0.9029 & 0.1307 & 0.1331 & 1.7968 \\
\hline 0.1280 & 0.1297 & 1.3564 & 0.1364 & 0.1401 & 2.7046 \\
\hline 0.1307 & 0.1331 & 1.8113 & 0.1424 & 0.1475 & 3.6185 \\
\hline 0.1335 & 0.1366 & 2.2675 & 0.1485 & 0.1553 & 4.5383 \\
\hline 0.1364 & 0.1401 & 2.7249 & 0.1550 & 0.1634 & 5.4639 \\
\hline 0.1393 & 0.1438 & 3.1836 & 0.1617 & 0.1720 & 6.3950 \\
\hline 0.1423 & 0.1475 & 3.6434 & 0.1686 & 0.1810 & 7.3315 \\
\hline 0.1454 & 0.1514 & 4.1043 & 0.1759 & 0.1905 & 8.2731 \\
\hline 0.1485 & 0.1553 & 4.5663 & 0.1835 & 0.2004 & 9.2195 \\
\hline 0.1517 & 0.1593 & 5.0292 & 0.1913 & 0.2108 & 10.1706 \\
\hline 0.1549 & 0.1634 & 5.4931 & 0.1995 & 0.2217 & 11.1258 \\
\hline 0.1582 & 0.1676 & 5.9578 & 0.2080 & 0.2331 & 12.0850 \\
\hline 0.1616 & 0.1719 & 6.4233 & 0.2168 & 0.2451 & 13.0478 \\
\hline & & & & & \\
\hline
\end{tabular}

Page | 7 


\begin{tabular}{|llllll|}
\hline 0.1650 & 0.1763 & 6.8895 & 0.2260 & 0.2577 & 14.0138 \\
\hline 0.1685 & 0.1809 & 7.3564 & 0.2355 & 0.2708 & 14.9825 \\
\hline 0.1720 & 0.1855 & 7.8237 & 0.2454 & 0.2846 & 15.9536 \\
\hline 0.1757 & 0.1902 & 8.2915 & 0.2557 & 0.2990 & 16.9265 \\
\hline 0.1793 & 0.1951 & 8.7597 & 0.2664 & 0.3141 & 17.9007 \\
\hline
\end{tabular}

\section{CONCLUSION}

Table.2.5: Evaluating the effect of varying the intrinsic growth rates together by $125 \%$ on biodiversity gain using ODE 45 numerical scheme.

\begin{tabular}{|ccccccc|}
\hline $\mathrm{C}_{\mathrm{b}}(\mathrm{t})$ & \multicolumn{2}{c|}{$\mathrm{C}_{\mathrm{bm}}(\mathrm{t}) \mathrm{BG}(\%)$} & $\mathrm{G}_{\mathrm{b}}(\mathrm{t})$ & \multicolumn{2}{c|}{$\mathrm{G}_{\mathrm{bm}}(\mathrm{t})$} & \multicolumn{2}{c|}{$\mathrm{BG}(\%)$} \\
\hline 0.1200 & 0.1200 & 0 & 0.1200 & 0.1200 & 0 \\
\hline 0.1226 & 0.1233 & 0.5637 & 0.1253 & 0.1267 & 1.1203 \\
\hline 0.1253 & 0.1267 & 1.1299 & 0.1307 & 0.1337 & 2.2510 \\
\hline 0.1280 & 0.1301 & 1.6984 & 0.1364 & 0.1410 & 3.3921 \\
\hline 0.1307 & 0.1337 & 2.2692 & 0.1424 & 0.1488 & 4.5433 \\
\hline 0.1335 & 0.1373 & 2.8423 & 0.1485 & 0.1570 & 5.7046 \\
\hline 0.1364 & 0.1411 & 3.4176 & 0.1550 & 0.1656 & 6.8757 \\
\hline 0.1393 & 0.1449 & 3.9951 & 0.1617 & 0.1747 & 8.0564 \\
\hline 0.1423 & 0.1488 & 4.5747 & 0.1686 & 0.1842 & 9.2464 \\
\hline 0.1454 & 0.1529 & 5.1563 & 0.1759 & 0.1943 & 10.4454 \\
\hline 0.1485 & 0.1570 & 5.7398 & 0.1835 & 0.2048 & 11.6532 \\
\hline 0.1517 & 0.1613 & 6.3253 & 0.1913 & 0.2159 & 12.8694 \\
\hline 0.1549 & 0.1656 & 6.9125 & 0.1995 & 0.2276 & 14.0935 \\
\hline 0.1582 & 0.1701 & 7.5014 & 0.2080 & 0.2399 & 15.3253 \\
\hline 0.1616 & 0.1746 & 8.0919 & 0.2168 & 0.2527 & 16.5641 \\
\hline 0.1650 & 0.1793 & 8.6839 & 0.2260 & 0.2662 & 17.8095 \\
\hline 0.1685 & 0.1841 & 9.2773 & 0.2355 & 0.2804 & 19.0609 \\
\hline 0.1720 & 0.1890 & 9.8720 & 0.2454 & 0.2953 & 20.3177 \\
\hline 0.1757 & 0.1940 & 10.4678 & 0.2557 & 0.3109 & 21.5792 \\
\hline 0.1793 & 0.1992 & 11.0647 & 0.2664 & 0.3273 & 22.8448 \\
\hline
\end{tabular}

By using ODE 45 we have found out that a biodiversity loss can be obtained due to a decreasing variation of the intrinsic growth rates together, whereas a dominant biodiversity gain can be obtained due to an increasing variation of the intrinsic growth rates together. On the basis of this analysis, the decreasing variation of the intrinsic growth rates together has generally indicated a decrease in the yields of these two crops, whereas an increasing variation of the same parameter values has indicated an improvement in the yields of both cowpea and groundnut. In this context, an alarming rate of biodiversity loss of these quantified magnitude are a strong signal on lower food production, endemic poverty and a weak sustainable development scenario, whereas a biodiversity gain has the potential to alleviate poverty and sustain development. These two components of biodiversity as predicted in this work have their policy implications.

This present numerical idea can be extended to examine the effects of varying the intra and inter competition coefficients together in our future investigation.

\section{REFERENCES}

[1] Atsu, J. U. \& Ekaka-a, E. N. (2017). Modeling the policy implications of biodiversity loss: A case study of the Cross River national park, south - south Nigeria. International Journal of Pure and Applied Science, Cambridge Research and Publications. vol 10 No. 1; pp 30-37.

[2] Atsu, J. U. \& Ekaka-a, E. N. (2017). Quantifying the impact of changing intrinsic growth rate on the biodiversity of the forest resource biomass: implications for the Cross River State forest resource at the Cross River National Park, South - South, Nigeria: African Scholar Journal of Pure and Applied Science, 7(1); 117 -130 .

[3] De Mazancourt, C., Isbell, F., Larocque, A., Berendse, F., De Luca, E., Grace,J.B etal. (2013). Predicting ecosystem stability from community composition and biodiversity. Ecology Letters,, DOI: 10.1111/ele.12088.

\begin{tabular}{|l|l|l|}
\hline $\begin{array}{l}\text { Statistical measure by } \\
\text { Table }\end{array}$ & $\begin{array}{l}\mathrm{BL}_{1} \\
\text { (Average) }\end{array}$ & $\begin{array}{l}\mathrm{BL}_{2} \\
\text { (Average) }\end{array}$ \\
\hline Table 1.1 & 16.801 & 29.5726 \\
\hline Table 1.2 & 15.9748 & 28.2803 \\
\hline Table 1.3 & 15.1369 & 26.9532 \\
\hline Table 1.4 & 14.2872 & 25.5902 \\
\hline Table 1.5 by & 1.0497 & 2.0550 \\
\hline $\begin{array}{l}\text { Statistical measure } \\
\text { Table }\end{array}$ & $\begin{array}{l}\mathrm{BG}_{1} \\
(\text { Average) }\end{array}$ & $($ Average) \\
\hline Table 2.1 & 1.0648 & 2.1134 \\
\hline Table 2.2 & 2.1448 & 4.2870 \\
\hline Table 2.3 & 3.2404 & 6.5226 \\
\hline Table 2.4 & 4.3518 & 8.8221 \\
\hline Table 2.5 & 5.4792 & 11.1876 \\
\hline
\end{tabular}

[4] Doak, D.F., Bigger, D., Harding, E.K., Marvier, M.A., O’Malley, R.E. \&Thomson, D. (1998). The statistical inevitability of stability-diversity relationships in community ecology. Am. Nat., 151, 264-276.

[5] Ernest, S.K.M. \& Brown, J.H. (2001).Homeostasis and compensation: the roleof species and resources in ecosystem stability. Ecology, 82, 2118-2132.Fowler, M.S. (2009). Increasing community size and connectance can increase stability in competitive communities. J. Theor. Biol., 258, 179-188.

[6] Fowler, M.S., Laakso, J., Kaitala, V., Ruokolainen, L. \& Ranta, E. (2012). Species dynamics alter community 
diversity-biomass stability relationships. Ecol. Lett.,15, 1387-1396.

[7] Gonzalez, A. \& Descamps-Julien, B. (2004). Population and community variability in randomly fluctuating environments. Oikos, 106, 105-116.

[8] Gonzalez, A. \& Loreau, M. (2009). The causes and consequences of compensatory dynamics in ecological communities. Annu. Rev. Ecol. Evol. Syst., 40, 393414.

[9] Grman, E., Lau, J.A., Donald, R., Schoolmaster, J. \& Gross, K.L. (2010).Mechanisms contributing to stability in ecosystem function depend on the environmental context. Ecol. Lett., 13, 1400-1410.

[10] Hector, A., Hautier, Y., Saner, P., Wacker, L., Bagchi, R., Joshi, J. et al. (2010).General stabilizing effects of plant diversity on grassland productivity through population asynchrony and over yielding. Ecology, 91, 2213-2220.

[11]Loreau, M.. \& de Mazancourt, C.. (2013). Biodiversity and ecosystem stability: a synthesis of underlying mechanisms. Ecol. Lett., DOI: 10.1111/ele.12073.

[12] Loreau, M. \& Hector, A. (2001). Partitioning selection and complementarity in biodiversity experiments. Nature, 412, 72-76.

[13] MacArthur, R. (1955). Fluctuations of Animal Populations, and a Measure of Community Stability. Ecology, 36, 533-536.

[14] Marquard, E., Weigelt, A., Roscher, C., Gubsch, M., Lipowsky, A. \& Schmid, B.(2009). Positive biodiversity-productivity relationship due to increased plant density. J. Ecol., 97, 696-704.

[15] May, R.M. (1973). Stability and complexity in model ecosystems. 2001, Princeton Landmarks in Biology edn. Princeton University Press, Princeton. McCann, K.S. (2000). The diversity-stability debate. Nature, 405, 228-233.

[16] McNaughton, S.J. (1977). Diversity and stability of ecological communities: a comment on the role of empiricism in ecology. Am. Nat., 111, 515-525.

[17] Mutshinda, C.M., O’Hara, R.B. \& Woiwod, I.P. (2009). What drives community dynamics? Proc. Biol. Sci., 276, 2923-2929.

[18] Proulx, R., Wirth, C., Voigt, W., Weigelt, A., Roscher, C., Attinger, S. et al.(2010). Diversity Promotes Temporal Stability across Levels of Ecosystem Organization in Experimental Grasslands. PLoS ONE, 5, e13382.

[19] Roscher, C., Weigelt, A., Proulx, R., Marquard, E., Schumacher, J., Weisser, W.W. et al. (2011). Identifying population- and community-level mechanisms of diversity-stability relationships in experimental grasslands. J. Ecol., 99, 1460-1469.

[20] Van Ruijven, J. \& Berendse, F. (2007). Contrasting effects of diversity on the temporal stability of plant populations. Oikos, 116, 1323-1330.

[21] Solomon, S., D. Qin, M. Manning, Z. Chen, M. Marquis, K.B. Averyt, M. Tignor and H.L. Miller (eds.)]. Cambridge University Press, Cambridge, United Kingdom and New York, NY, USA, 996pp.

[22] Rahmstorf, S., Cazenave, A., Church, J.A., Hansen, J.E., Keeling, R.F., Parker, D.E., and R.C.J. Somerville, 2007: Recent climate observations compared to projections. Science 316 (5825):709-709.

[23] Domingues, C.M, Church, J.A., White, N.J., Gleckler, P.J, Wijffels, S.E., Barker, P.M. and J.R.Dunn, 2008:. Improved estimates of upper-ocean warming and multidecadal sea-level rise. Nature 453:1090-1094. 